UCRL- 98174

PREPRINT

\title{
A GENERAL PURPOSE CALIBRATION FACILITY FOR USE IN Y-RAY ASTRONOMY
}

H. H. Craig, C. J. Hailey, R. I. Morales

Laboratory for Experimental Astrophysics

University of Caifornia

Lawrence Livermore National Laboratory

Livermore, California 94550

S. II. Kahn, B. H. Hargelin

Department of Physics

University of California

Berkeley, California 94720

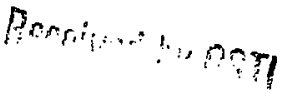

$$
\begin{aligned}
& \text { SEF }=1,5
\end{aligned}
$$

\section{PHYSICS/V-OIVISION}

This paper was prepared for submittal to:

SOCIETY FOR PHOTO-OPTICAL INSTRUPENT ENGINEERING (SPIE)

San Diego, CA, August 15-18, 1988

August 11, 1988

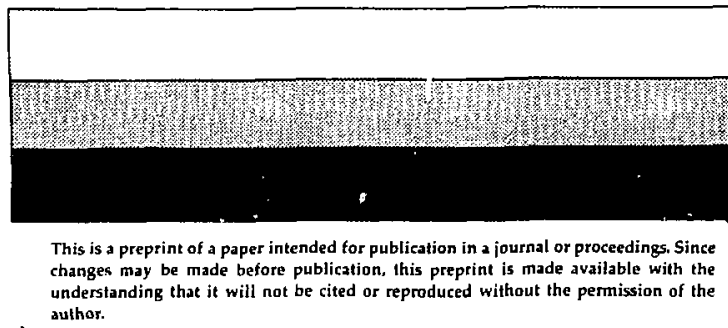
author.

\section{DISCLAIMER}

This repart was prepared as an aceorunt of work speinsured by an agency of the United States Government Nesther the lInited States Guvernment nor any agency theteof, nas any of their

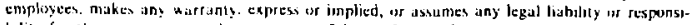
bulity for the - ewraty. completeriess, or use[ulness af any infurmaluon, appurutus, product, ur proces discined, uf tepresents that ts use would not infringe privately owned tights Refer. ence hefe:n wo any specafic cornmetcial preduel, provess, ur service by trade name. trademark. manufaclurer. of utherwise docs not necessasily constivitu us imply its emotonement, recommendateron, es favuring by the United States (jovernment of any agency thereof The views and apininas af uuthors expressed hercin do not nescessarily slate or reflect those of the Linited States Kuvernment of any agency thereol. 
A general purpose calibration facility for use in x-ray astronomy

\author{
W. W. Craigt, C. S. Haileyt, R. I. Morales \\ Lawtence Livermore Natioral Laboratory \\ P.O. Bax 808 L-401 \\ Livermare, California 94550 \\ S. M. Kahnt, B. J. Wargelint \\ Deparment of Physics \& Space Sciences Laboratory \\ University of Califomia, Berkeley \\ Berkeley, California 94720

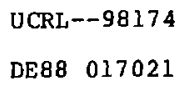

\begin{abstract}
An $x$-ray calibration facility for use in the $0.2-25 \mathrm{keV}$ region is described. The facility employs several types of sfecially modified sources and detectors to produce and detect both line and continuum radiation in this energy range. We describe an inexpensive commercial $x$-ray source which has been modified for efficient high intensity operation as well as production of $x$-mys up $025 \mathrm{keV}$. We also describe a system that utilizes multilayer mirrors alone or in a double Bragg geometry to select an energy bandpass. This system is controlled by a microcomputer which translates and rotates the multilayers 10 provide an easily selectable monochromatic beam with good resolution over a broad energy range. A long focal length Kirkparick-Baez thirror pair has been coupled wo a pivoting bean line in order to accurately chancterize gratings for use in soft $x$-ray astronomy. The beam line is scanned through the various grating orders. All aspects of the facility incorporate a high degree of flexibility so that a wide variety of culibralions can be easily performed.
\end{abstract}

\title{
1. INTRODUCTON
}

A calibraion facility should be able to perform accurare measurements on virtually any piece of appararus in its particular range of energies We have attempted 10 construct a facility that can measure the quantum efficiency of a new detector, the transmission of a thin window, or the efficiency of an $x$-ray optic with relative ease. As the facility perfomis calibrations in support of $x$-ray astronomy, the primary energy ranges are fixed by the regions of the $x$-ray spectrum that are most likely $\omega$ be of interest. At the lower energy end of the $x$-ray spectrum, missions now on the drawing board use low to moderate resolution dispersive elements to investigate the energy range from 200-2000 eV. This soft $x$-ray region includes the $K$-shell transitions of Carbon, Nitrogen and Oxygen as well as the L-shell transitions of Iron. Spectral features due to these elements have been identified in many $x$-ray sources detected by earlier $x$-ray sarellites. At the higher energies, solid stare detectors as well as the mure traditional crystals and scintillation devices will be used.

Ongoing work at the Laboratory for Experimental Astrophysics ar LLNL includes development of an $x$-ray bolometer and hard $x$-my imaging detectors as well as low blaze angle reffection gratings. The facility now in operation was designed to support the needs of all of these projects while also handling routine calibratich. Nork. We will describe the sources that have been developed to supply the $x$-rays, a multi-use chamber utilizing the yarious sources, a monochromator to provide wide band continuous coverage, ano a special long bearn facility used in the development of $x$-ray optics.

\section{SOURCES}

There are numerous ways of producing $x$-rays for calibration puposes. Swage rings, for example, provide a tunable, monochromatic, high intensity beam. The rings are, however, quite bulky and expensive, restricting their use $\mathrm{t}$ a few, specially equipped locations. Radioactive sources are usefil for producing line radiation and are quile 
portable but are restricted by the relative lack of available lines, particularly in the sub- 5 xeV range. Various other more exotic sources exist; in general they require fairly complex mechanisms to produce the $x$-rays.

In practice most taboratory $x$-ray sources consist of a hot filament that produces electrons which are then accelerated by an applied potential io an anude of the desired target material. These sources are compact, reliable and inexpensive ant produce a reasonable $x$-ray flux. Their spectra are easily calculabie ${ }^{l}$ and various experimental data exist on the feaures and incensity wo be expected ${ }^{2}$.

In an electron impact source boch characteristic line and continuum radiation is emitted. The continumm is mostly bremstrahiung and for a thick target is of the form

$$
I(v)=C Z\left(r-v_{0}\right)
$$

where $\mathrm{C}$ is a constant detemined erıpirically ${ }^{3}$. This continum radiation makes it difficult to obtain a sufficiendy monochromatic beam to perform :adibrations. A judicious selection of anode mateizal and edge filters makes most calibrations possible if a characteristic line may be generated in the energy region of interest. Some calibrations, however, require a cleaner beam than filters can provide.

To more nearly monochromatize the bean produced by an electron impact source, the initial $x$-rays may be aimed at a secondary target, producing flouresrence photons at the characteristic line energies of the secondary material. This method requires a large flux of primary photons to produce useful numbers of flourescent photons. Monochromators may also be used to select out sections of the continuum, providing a continuous spectrum. Either method requires a high input intensity to produce useable flux.

An electron impact source without active cooling can achieve no intensity higher than about $10^{10} \gamma_{s}$ ster ${ }^{-1} s^{-1}$ integrated over an cmission line. In a multi-purpose system the sources and detectors tend to be loosely coupled to allow for various size samples or insorumentation, the resultant loss due wo apertures and collimators can be 3-5 orders or more in intensity. If a monochromatic beam is needed, then any mechanism used to strip unwanted spectral fearures will reduce the flux by $1-3$ orders of magnitude more. Calibrations of inefficient detectars or dispersive elements are difficult and time consuming with such a source.

Eloctron impact sources are typically less than $1 \%$ efficient, most of the energy in the electron beam being thermally dissigated. Thus to obtain a large flux the anote must be cooled or rotated to always present a fresh surface to the electron beam. In order to obtain the $10^{13}-10^{14} \mathrm{~V}_{\mathrm{s}} \mathrm{ster}^{-1} \mathrm{~s}^{-1}$ needed to perform calibrations casily, the primary source for the flourescent photons, or the input to the monochromator must be actively cooled. Many cooling methods have been repore $\mathrm{d}^{4}$; the rotaling anode prirciple yields the most flux but is quite complex as it involves a high speed rotational vacuum seal. The water cooled source is far easier to construct and can easily produce produce $-10^{14} \gamma_{s}$ ster $^{-1} s^{-1}$ per kilowall of input power.

\subsection{Facility $X$-ray sources}

Our facility utilizes two water cooled sources, one a direct emitter, the other an electron impact source coupled to flourescent targets. The flourescent source is modeled after the device constructed by Henke 2 . As shown to the side in figure 2 the $x$-ray tube illuminates a carousel of various flourescence argets which can be rotated into positon. producing characteristic line emission. The $x$-rays from the direct source are not collimated or focussed, yielding a rather diffuse emission from the arget with the emission zone limited only by the size of the target. The outpu from this source is limited in energy by the available target materials as well as by the upper energy limits set by the anode material of the direct source. The primary Henke tube produces a broadband spectrum that may be used directly if needed. The available flux is limited however, as the emission comes from a large $1.5 \mathrm{~cm}^{2}$ area, thus any collimators or apertures cut down the flux significanlly.

To produce a more nearly pointlike, high brilliance source of $x$-rays over a wide range of energy we modified a commercial $x$-ray source sold by the J.E. Manson Co. This source as supplied was passively cooled and thus could support input power of only a few watls, resulting in fewer than $10^{10} \gamma \mathrm{s}$ ster ${ }^{-1} \mathrm{~s}^{-1}$ Irom a spot size on order 200 microns in diameter. We have used these sources frequenty in the pase They are simple to operate and maintain. and are compact enough to fit through a standard 2.75 inch vacuum inlet making them easy to adapt to various uses. As shown in figure 1 we modified the source by replacing the anode and anode feedthrough by a bored out shaft which allows water to pass within $4 \mathrm{~mm}$ of the anode surface. The staft is scparated from the walls by 
ceramic (Macor) insulators allowing operation up to $25 \mathrm{keV}$. The removable anodes are sealed to the shaft using an Indium O-ring. The original design utilized Viton $O$-rings to seal the anode to the shaft and tended to produce excessive cabon buildup on the anode. The carbon buildup is an intrinsic problem with this type of source and may only be avoided by careful cleaning and adequate pumping.

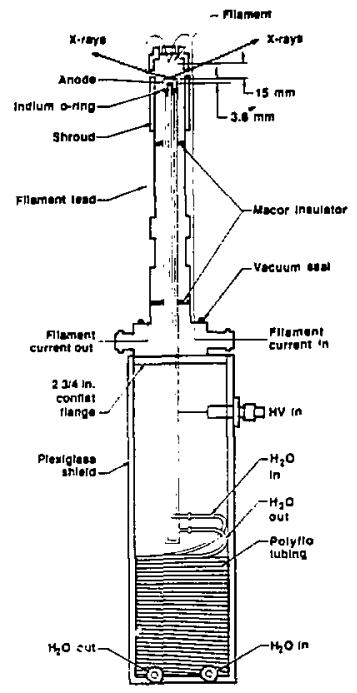

Figure 1 - A modified $x$-ray source utilizing active water cooling. The source extends 200 $\mathrm{mm}$ past the vacuum seal into the source chamber. $\mathrm{X}$-rays are emitted in two beams seperated by 120 degrees. The anode is easily interchanged with several materials currently available.

Cooling water is brought in and out through a long coiled section of plastic tubing. The tubing, high voltage connector, and filament leads are protected by a plexiglass shield. The area around the anode and filament is covered by an aluminum shroud that limits the $x$-ray beam to selected beam paths. The apertures in the shroud car be easily changed in both size and location to fit the specific needs of a calibration project Generally they are on opposite sides of the shroud, at an angle of 30-45 degrees with respect to the plane containing the anode. One bearn is used for calibration purposes, the other is directed at a reference detector that monitors source stability. The two beam lines have been measured to be equivalent to within $2 \%$.

This source is inexpensive and very reliable, allowing the generation of a significant amount of $x$-rays over a wide energy range. This source has a brightness of $10^{13}-10^{14} \gamma^{s}$ ster $-1 s^{-1}$ in the emission lines with $\mathrm{Al}$ and $\mathrm{Cu}$ anodes from a $1 \mathrm{~mm}^{2}$ spot size. Typically the source is operated with less than 1 kilowat of input power. Graphited copper can be used to produce Carbon $\mathrm{Ka}$ radiation at a slighly higher flux level.

We also use a multi-anode source supplied by the J.E. Mansen Co. that incorporates the same design as the single anode version as described above. This source contins six anodes on a carousel, all connected to a common high voltage. The carousel can be rotated while under vacuum, enabling simple, rapid changes in the $x$-ray spectrum being used to do a calibration. This source is not cooled, limiting its input power to a few warts. The source produces wo beam lines, separated by 90 degrees, as shown in Figure 2. 


\section{DETECTORS}

Two main types of detectors have been used in our calibration work. For most tasks we have used flow type proporional counters. These counters generally are used at atmospheric pressure for work above about $900 \mathrm{eV}$, with a pressure regulating system reducing the pressure down to a limit of a few torr for lower energy work. The windows are either thin beryllium or .8um polypropylene stretched over a $70 \%$ transmissive nickel grid. The counters are generally mounted inside the vacuuni chambers on either remocely controlled transiation tables or on rotary feedthroughs so that they may be scanned while under vacuum. For work requiring higher resolution, we have adapted a windowless lithium- drifted silicon (SiLi) detector to a bellows that allows it to be attached to a chamber and scanned over a limited range ( $+/-10$ degrees). The SiLi detector has a typical resolution E/AE of 30 at $6 \mathrm{keV}$, and can resolve Boron and Carbon Ka's at the lower energies.

\section{CALIBRATION SETUP.}

The various sources and detectors are combined with translation and rotation stages in a multi-use chamber as shown in Gigure 2. As depicted, the chamber is configured to measure the efficiency of a mulilayer. The multilayer can be moved out of the bean for an $l_{0}$ measurement, then ceturned into the path of the beam and rocated to the desired angle $\theta$.

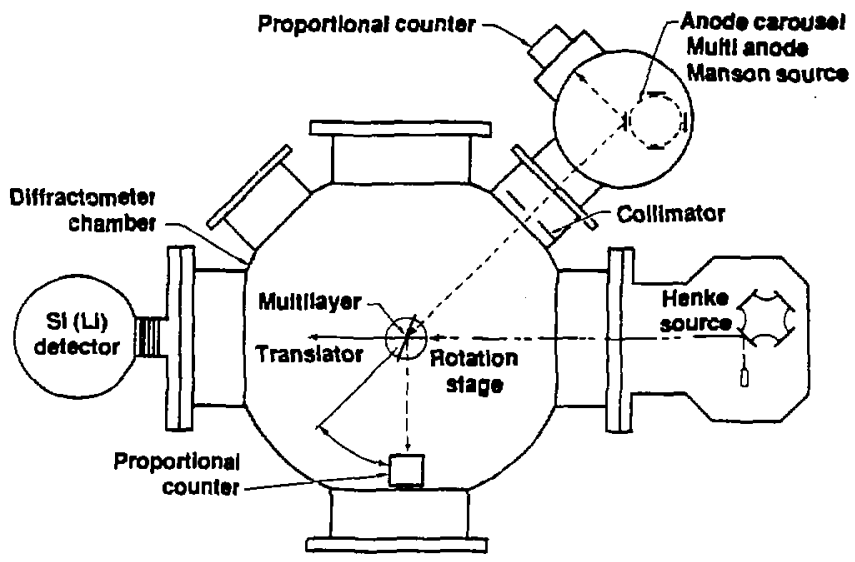

Figure 2 - Multi-use calibration chamber in a typical configuration. The multilayer may be illuminated by either of the two sources. Diffracted $x$-rays can be viewed with either a scanning proportional counter or a SiLi detector mounted on a bellows allowing 20 degrees of angular motion. 
The proportional counter is then roiated through $2 \theta$ and the measurement taken. The multilayer can also be used as a predisperser with its outpul beam directed at the filter or crystal to be calibrated. The chamber itself is ${ }^{-} 40 \mathrm{~cm}$ in diameter and the proportional counter is free to rotate through -320 degrees. The large available area and dual sources enable a wide variety of calibrations to be made.

\section{DOUBLE MULTTLAYER MONOCHROMATOR}

Although the combination of flourescent and direct electron impact sources serves many purposes, the wavelengths available are, in general, limited $\omega$ a few lines, particularly below about I keV. Providine. from these souces, a continuous specirum throughout the energy ranges of interest requires a dispersive monochromator. To maintain reasonable throughput while maintaining moderate resolution, synthetic multilayers are the best choice as dispersing elements. For flexibility and ease of use, fixed entrance and exit slits are desirable.

A design of this son is not paricularly novel. Barbee 5 , for example, has described a double multilayes monochromator that maintrins this relationship by using a mulhilayer pair with a layer pair spacing that is graded along the length of the multilayer. This son of system can maintain a fxed exit slit by transtaing one mirror over a limited range.

The fixed entrance and exit slit relationship for various energies can also be maintained by using identical multilayer pairs in a parallel configuration. Both multilayers must be rotated and one translated along the beam path. This arrangement has been used in double crystal spectrometers ${ }^{6}$. By simply following the corrected Bragg formula

$$
m \lambda=2 d \sin (\theta)\left(1-8 / \sin ^{2} \theta_{0}\right)
$$

where $\delta=1-n_{0}$ with $n_{0}$ being the refractive index of the material, the multilayer elements can be rotated and the second mulolayer translated to the proper position to maintain the fixed exit bean.

This arrangement is shown in Figure 3. The entrance slit is auached w a front bafle plate, which when com: bincd with a back baffle plate, totally excludes all light except that which is diffracted, or specularly reflected, off the nulcilayer mirrors. The stages are driven by small de motors with integral encoders. The motors are controlled by an IBM PC through an RS-232 compacible motor contsoller. The vertical distance berween the two mirrors is mechanically fixed. The horizontal distance is determined during initial alignment. After establishing the correct angles by calibrating off of a known spectrum, i.e. locating a strong single line, the monochromator can maintain the correct energy, to within a few eV, throughout a scan. The mirors can be moved independenty to allow final tine adjustment 10 maximize flux at a given wavelength.

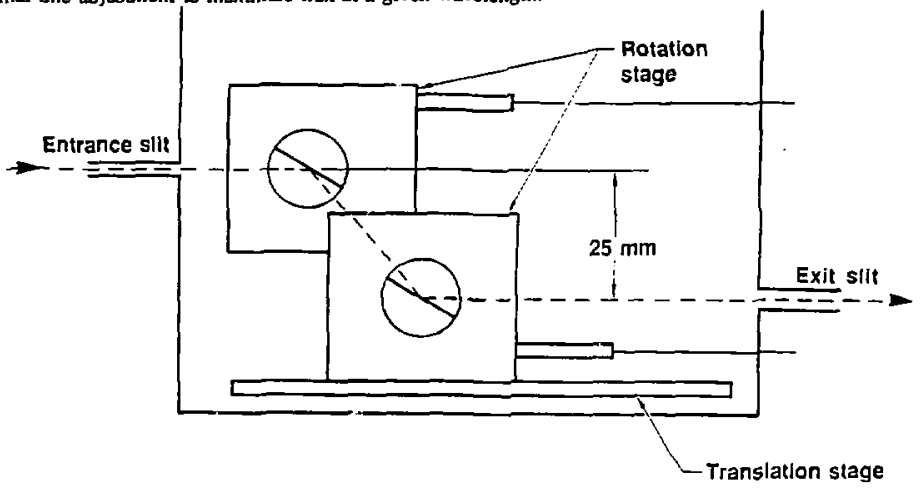

Figure 3 - Schematic of a double muliblayer monochromawor. The lower rotation stage transtates along the beam direction to kecp the exit beam at the same position as the mirrors are rotated. 
There are three sets of multilayers currently available. One pair with a 2d spacing of 120 angstrom, consisting of layer pairs of tungsten and silicon, covers an energy range from $-200 \mathrm{LO} 500 \mathrm{eV}$. A second pair with a $2 \mathrm{~d}$ spacing of 60 angstroms and layer pairs of tungsten and carbon, covers the 500 to $1100 \mathrm{eV}$ range. For the range above I keV, a pair of tungsten/carbon mirrors with a $2 \mathrm{~d}$ spacing of 30 angstroms will be used. The mirrors are approximately 30 by $80 \mathrm{~mm}$ in size and are deposited on a $.25 \mathrm{~mm}$ silicon wafer. The mululayers were purchased from Ovonics Synthetic Materials.

The performance of multilayers, i.e. their refiectivity and resolution, can be easily calculated?. Using the parameters for the multilayer as supplied by the vendor, and veribed in our own sebup, we calculaced an expected resolution, $\lambda \Delta \lambda-80$ at $900 \mathrm{eV}$, as compared to an expected resolution of about 50 for a single cuuluilayer of this type at this energy. As shown in figure 4, the FWHM of the $C u$ La line at $929 \mathrm{eV}$ is $-16 \mathrm{eV}$, yielding a $\lambda \Delta \lambda$ of $>60$. The small peak at $950 \mathrm{eV}$ is the $\mathrm{Cu} L \beta_{1}$ line. For these parameters the beam is only $60 \%$ o polarizeci. As the angle increases toward 45 degrees, the beam bocomes more nearly polarized. If the $20=30$ mirrors were used in the above case, the bcan would be $90 \%$ o polarized.

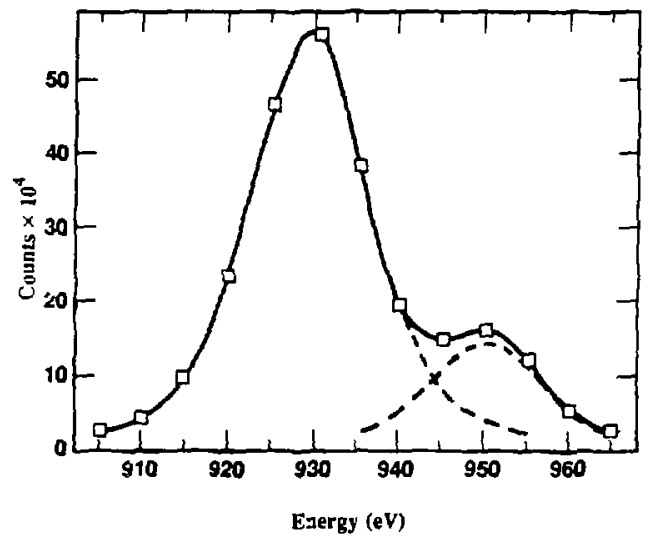

Figure 4 - Result of a scan by the double mulitayer monochromator while illuminated by an electron impact source with $C u$ anode. The $C u L \alpha$ line is at $929 \mathrm{eV}$. The smaller feature is $C u L \beta_{1}$ ar $950 \mathrm{eV}$. The dashed lines are gaussian fits to the peaks. Exhibited resolution is $h / \Delta \sim 60$.

To be useful the monochromator must be efficient enough not only wo pull out a characteristic line, but also must be able to separate sections of the continuum out Cohen and Deslattes ${ }^{8}$ calculate the amount of lux available in a bremstrahling conjinuum.

$$
N_{B}=V i Z\left(1-E / E_{0}\right) \gamma s s^{-1} m^{-2} \text { per } 10^{-3} \Delta E / E \text {. }
$$

$V$ is the accelesating voleage and $i$ the current $E_{Q}=V^{\prime} e$. The water cooled source described in section 2 is typically cperated $a$ about $6.5 \mathrm{kV}$ and $90 \mathrm{~mA}$. With a copper anodc, a total combined efficiency off the mirors of $10 \%$, and including the geometric factors, equation (3) predicts about $5 \times 10^{3} \gamma^{\prime} \mathrm{s}^{-1}$ per $16 \mathrm{eV}$ energy interval at $800 \mathrm{eV}$. We measured a coum rate of $3 \times 10^{3} \gamma s^{-1}$ in the $16 \mathrm{eV}$ interval around 800 . This count ate, concained in a very slowly diverging beam, is sufficient to perform most calibrations. The performance of the system at higher energies with the multilayer pairs with a $2 \mathrm{~d}$ of 30 angstroms has not been tested but is expected to be roughly comparable. 


\section{LONG BEAM LINE ASSEMBLY}

The next generation of $x$-ray observatories will include high throughput missions that couple moderate resolution telescopes (1 arcmin) with reflection gratings to provide medium resolution spectroscopic information in the 200$2000 \mathrm{cV}$ region?. These reflection gratings are likely to be characterized by a low blaze angle ( $<1$ degree), and a varied line spacing to maintain resolution in a converging beam ${ }^{10}$. Such low blaze angles are technically difficult to produce, and in practice many such gratings need to be tested before acceptable samples are found. To properly test the resolving power of the gratings, they must be placed in a converging beam with a long focus approximating that of the eventual $x$-ray telescope focus.

To test sample gratings intended for use in such an experiment we have configured existing $x$-ray calibration facility equipment into a 8 meter facility as shown in figure 5. This facility uses either an actively or passively cooled electron impact source to produce $x$-rays. The converging bean is produced by a Kirkparick-Bacz mirror pair placed in close proximity w cie source. These mirrors employ a unique dual lever am tension arrangement to bend gold coated foat glass in a nearly perfect ellipse 11 . The mirrors focus the $x$-ray source on the detector, in the smalles chamber, some 7.5 meters away. Because of their proximity wo the source, the mirrors act as magnifiers, requiring the use of a pinhole in from of the $x$-ray source to limit the size of the focal spot 10 a maximum of one half the size of the detector window. The mirrors are focussed under vacuum, in the $x$-ray, through use of remove controlled đc motors.

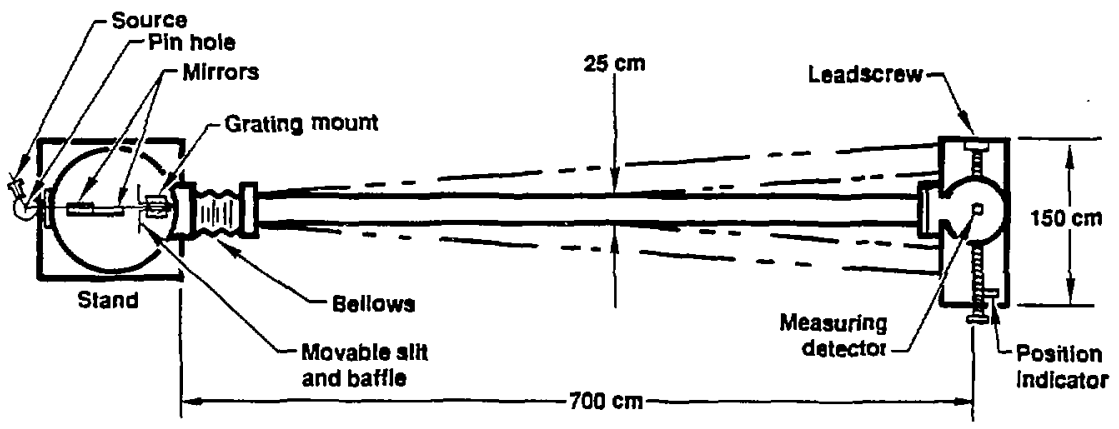

Figure 5 - The long beam facility for measuring the performance of varied line space reflection gratings. The $x$ rays from the source at the left are focussed by the two mirrors in a Kirkpatrick-Baez configuration, the grating then disperses the orders where they are scanned by moving the encire beam line with the leadscrew at the far right.

With the $x$-rajy beam focussed, a slit is placed betwein the mirors and the test grating so that various sections of the grating can be illuminated independenty. The grating is mounted on a combination rotation and translation stage and is moved inco the bearn and rotated to its proper angie. The spectral orders are dispersed along a line perpindicular to the grating surface. The entire beam line pivots on a bellows, allowing the spectrum to be scanned through several orders. A Jow backlash leadserew and position indicator on the detector chamber end give angular accuracies of beuer than 10 arcsecunds, much beuer than that required by the grating or telescope parameters. Several graing samples and calibrated mirrors have been tested in the facility with results consistent with other reponed figures 12 . 


\section{CONCLUSION}

We have constructed a very fiexible calibration facility that altows us to perform accurate measurements on a wide variety of optical elements, filters and detectors. The facility can operate in a continuous fashion from $200 \mathrm{eV}$ to more than $2000 \mathrm{eV}$ with the capability of calibrations up to $25 \mathrm{keV}$. This facility curtently supports several ongoing $x$-ray astronomy experiments as well as performing routine calibration wotk. We have constructed a double multilayer monochromator that operales with near theoretical resolution over a wide energy range. A long beam facility to lest low blaze angle, varied line space gratings is in operazion and producing useful results.

\section{ACKNOWLEDGEMENTS}

We would like to thank S. Compton for his work on the long beam facitity and T. Stack for his help with the water cooled $x$-ray source. This work was performed under the auspices of the U.S. Department of Energy by Lawrence Livemire National Laboratory under contract No. W-7405-Eng-48.

\section{REFEBENCES}

1. D. B. Brown and D. J. Nagel, "Calculation of Spectra from Electron Impact X-Ray Sources "

in, Low Energy X-Ray Diagnostics David Atwood, ed., AlP Conference Proceeding 75, (1981) 253-260.

2. B.L. Henke in Proceedings, ERDA X-and Gamma-Ray Symp Ann Arbor, M, May 19-21,1976 Conference 760539. (1976) 36-39.

3. H. Kulenkampfi, Am. FIAT Rev. German Sci. 12 Ch.3 127 (1950).

4. M. Yoshimatsu and S. Kozaki, "High Brilliance X-Ray Sources," in Topics in Applied Physics Vol. 22, (Springer-Verlag, Berlin), (977,Chapter 2.

5. T.W. Barbee, Jr., "Multilayers for X-ray Optical Applications" in X-Ray MicrosconY ed. G. Schmahl and D. Rudolph (Springer-Verlar, Series in Optical Sciences Volume 43, Berlin 1984) 144-162.

6. H. Kawata and M. Ando, "A Double Crystal Monochromator for the Photon Factory Wiggler Beamline", Nuclear Instouments and Methods in Physics Research A246 (1986), 368-372.

7. J.H. Underwood Jr. and T.W. Barbee, Jr., "Synthetic Multilayers as Bragg Diffractors for X-Rays and Exureme Ultraviolet: Calculations of Performance", in Low Energy X-Ray Diagnostics David Atrwood ed., AIP Conference Procecdings 75, (1981) 170-178.

8. G.G. Cohen and R.D. Deslattes, "Application of a High Intensity Laboratory X-Ray Source to EXAFS Spectroscopy." Nucl. Insts. and Methods 193 (1982) 33-39.

9. ESA SP-1097,"The High Throughput X-ray Spectroscopy Mission", March (1988).

10. S. M. Kahn and M.C. Heurick, in, Proc. of ESA Workshop on Cosmic Ray Spectroscopy Mission, Lygby, Denmark, ESA SP-239, (1985), 237.

11. The mirrors were obtained from Acton Research Corp, Acton, Mass. Model AMA-250.

12. A.J.F den Boggende et. al., these proceedings (1988). 